\title{
Awareness of retinopathy of prematurity among pediatricians in West Bank, Palestine: a descriptive study
}

\author{
Mohammad T. Akkawi ${ }^{1}$, Jamal A. S. Qaddumi2 ${ }^{2}$, Hala R. M. Issa² and Liana J. K. Yaseen²
}

\begin{abstract}
Background: Retinopathy of prematurity (ROP) is a disorder of the developing retina of preterm infants due to defective vasculogenesis. The aim of the study was to analyze the level of awareness, knowledge, attitude and practice of pediatricians about ROP in the West Bank, Palestine.
\end{abstract}

Methods: A questionnaire was designed on the knowledge, attitude, and practice (KAP) pattern. The questionnaire included questions about pediatrician's educational and practicing profile, knowledge of screening guidelines, risk factors for ROP, referral facilities and barriers for referral. The questionnaire was given to70 practicing specialists and residents in hospitals having neonatal intensive care units in the West Bank, Palestine. It was a self-administered questionnaire, collected between November 2016 and February 2017.

Results: A total of 70 pediatricians from 11 different hospitals without ROP screening service participated in the study. The mean age of the participants was $33.04 \pm 7.74$. Of which, $62.9 \%$ were males and $37.1 \%$ were females. Fifty-nine (84.3\%) answered that ROP is preventable, while 11 (15.7\%) responded that ROP is not preventable. Nine (12.9\%) pediatricians had no idea as to which part of the eye is affected in ROP. Among the participants, 29 (41.4\%) did not know when ROP screening should be started. Sixty-three (90\%) pediatricians were sure that ROP is treatable. Regarding barriers for ROP screening, 'ophthalmologist not available' reason was expressed by $37.1 \%$ (26/70), 'discharge person not writing' by 20\% (14/70) and 'parents not agreeing' by 18.6\% (13/70) of the participants. Knowledge on the use of laser as a treatment modality of ROP was shown by 39 (55.7\%) participants, and the use of anti-VEGF was shown by 6 (8.6\%) participants, whereas 25 (35.7\%) of the participants didn't know about the treatment modalities.

Conclusion: The study findings suggest that a large majority of pediatricians were aware of ROP as a preventable disease, but had less information about ROP screening guidelines and service delivery. The study suggests the need to increase the awareness of pediatricians by dissemination of information about ROP and creating a close coordination between them and ophthalmologists to address barriers for service delivery in Palestine.

Keywords: Awareness, Pediatricians, Retinopathy of prematurity

\footnotetext{
* Correspondence: Jamal9877@najah.edu

${ }^{2}$ An-Najah National University, Nablus, Palestine

Full list of author information is available at the end of the article
}

(c) The Author(s). 2018 Open Access This article is distributed under the terms of the Creative Commons Attribution 4.0 International License (http://creativecommons.org/licenses/by/4.0/), which permits unrestricted use, distribution, and reproduction in any medium, provided you give appropriate credit to the original author(s) and the source, provide a link to the Creative Commons license, and indicate if changes were made. The Creative Commons Public Domain Dedication waiver (http://creativecommons.org/publicdomain/zero/1.0/) applies to the data made available in this article, unless otherwise stated. 


\section{Background}

Retinopathy of prematurity (ROP), previously called retrolental fibroplasias, is a vasoproliferative disorder of the retina which occurs principally in premature children due to defective vasculogenesis as a result of exposure to risk factors [1]. It mainly affects premature babies born at or before 32 weeks and weighing $1500 \mathrm{~g}$ or less at birth [2]. However, larger and more mature infants can develop severe ROP in countries with low/ moderate levels of development compared with highly developed countries [3]. Several risk factors have been found to be associated with the development of ROP. The most identified are low gestational age, low birth weight, poor postnatal growth, inadequately administered oxygen supplementation, respiratory distress and blood transfusion. Most studies show the most significant factors to be low gestational age and low birth weight $[4,5]$. A significant improvement of the standards in neonatal intensive care units (NICUs) and perinatal care has increased the survival rate for the premature babies over the last decades. Consequently, the incidence of ROP has increased in parallel [6].

Retinal examination in preterm infants should be performed by an ophthalmologist with a sufficient knowledge and experience in order to identify accurately the location and sequential retinal changes of ROP [7]: Screening would be undertaken in the neonatal unit for babies who are still in-patients. Discharged babies can be examined in the neonatal unit during follow up, or in eye departments. Revised guidelines for screening have been suggested by the American Academy of Pediatrics, the American Association for Pediatric Ophthalmology and Strabismus and the American Academy of Ophthalmology. Those recommend screening for ROP in all infants with birth weight $<1500 \mathrm{~g}$ or gestational age of 32 weeks or less [8]. In contrast, the British Association for Perinatal Medicine and the College of Ophthalmologists recommend screening only infants $<1500 \mathrm{~g}$ at birth [9]. Timing of the initial examination is based on both postmenstrual age (PMA) and chronological age (CA) and is undertaken to detect $99 \%$ of infants at risk of a poor visual outcome. The first examination is conducted between four and nine weeks CA, depending on PMA at birth. Subsequent studies have confirmed the efficacy of conducting the first examination at four weeks CA in more mature infants [10]. These criteria only apply to these high income settings, wider criteria are used in less well resourced settings and less developed countries to ensure that all babies at risk are examined [3].

The current gold standard in treating sever, Type 1 ROP is still panretinal laser photocoagulation. However, ongoing studies in premature infants have been investigating the safety and efficacy of antiangiogenic therapies, especially anti-VEGF drugs. This seems to provide valuable and encouraging information for ROP treatment in the near future, though the long-term ocular and systematic safety of Anti-VEGF agents is not yet known [11]. A study was done in South India showed that awareness of ROP is poor among pediatricians. The same study mentioned that reports from other developing countries like China, Thailand and Vietnam also show a similar trend [12]. A study was done in 2013 in Nigeria to determine the level of awareness of the screening protocols for ROP among pediatricians, concluded that although majority of pediatricians are aware of ROP, they are poorly informed on the management and screening of the condition, and they need to be educated to be aware in order to prevent this treatable cause of blindness in children [13]. Another study was conducted in India 2011 to evaluate the prevailing practices for screening and referral scheme among Indian pediatricians for ROP concluded that only $14.5 \%$ were following international recommendations for ROP referral and that the screening remains poor due to the non-availability of trained ophthalmologists as well as inconsistent screening guidelines [14].

Retinopathy of prematurity is emerging as an important cause of avoidable blindness in both developed and developing countries [15]. Proper screening and referral practices will help saving sight for a more productive life. This is vital as number of blind years in a child increases the burden of care upon the family affected and the society as a whole $[16,17]$. To the best of our knowledge, no studies were done in Palestine concerning ROP screening or awareness among pediatricians. This study was conducted to assess knowledge, attitude and practice patterns (KAP) of pediatricians about ROP.

\section{Methods}

The study was a cross-sectional non-interventional descriptive study. It was conducted in eleven different Palestinian hospitals (private and governmental) in the West Bank that contain neonatal intensive care units (NICUs) and have no ophthalmology departments. Pediatricians usually consult a specialized ophthalmologist from outside to screen cases with high risk for ROP, any intervention was undergone in a specialized hospital. We invited seventy pediatricians (specialists and residents) who are in direct contact with neonatal care who all participated in the study. The data were collected between November 2016 and February 2017. A self-administered semi-structured questionnaire was formed on the knowledge, attitude and practice (KAP) pattern. It was gathered and modified from other questionnaires of similar published researches to assess knowledge, screening, referral barriers and treatment of ROP [12].

The study was approved by An-Najah National University IRB committee. Also, informed consent was taken from 
each participant, explained clearly that participants' privacy was insured.

\section{Results}

Seventy pediatricians participated in the study. The demographic details are given in Table 1 . The mean age of the participants was $33.04+-7.74$ years, range 25 to 59 years. $44(62.5 \%)$ were males. There were 31 (44.3\%) qualified pediatricians, while residents in training were 39 (55.7\%). 16 (22.6\%) had been practicing for more than 10 years, $14(19.9 \%)$ had about 5 to 10 years of practice experience and $40(57.2 \%)$ had less than 5 years practice experience. Most of the respondents, 67(95.7\%) were governmentally employed.

Fifty nine $(84.3 \%)$ pediatricians said that ROP is preventable, $3(4.3 \%)$ responded that ROP is not preventable, and 8 (11.4\%) did not know. Twenty-four (34.3\%) participants thought that blindness due to ROP is reversible, $35(50.0 \%)$ said it is irreversible, whereas 11 (15.7\%) did not know (Table 2).

Regarding ROP identification, 61 (87.1\%) mentioned that it is identified by examining the retina and 9 (12.9\%) did not know. Table 2 shows pediatrician's awareness about who performs screening for ROP.

Table 3 shows percentages of pediatricians with respect to their idea on the period of first eye test for ROP screening, that is, when to refer to ophthalmologist for ROP screening.

Referral for ROP screening was routinely undertaken by $67(95.7 \%)$ respondents. The major two methods of referral among those were writing a discharge slip (60\%) or informing the parents verbally (42.8\%). The main reason given for not screening for ROP was the unavailability of an ophthalmologist in the same hospital among 26 (37.1\%). Other contributing reason had to do with the discharging doctor not writing a screening referral order in 14 participants (20\%).

Sixty-three (90.0\%) pediatricians were sure that ROP is treatable, while $7(10.0 \%)$ thought the opposite. Knowledge on the use of laser or anti-VEGF injection as a treatment modality of ROP was recorded in 39 (55.7\%)

Table 1 Demographic details of the participants

\begin{tabular}{llll}
\hline Variable & Category & Frequency & Percent \\
\hline Gender & Male & 44 & 62.5 \\
& Female & 26 & 37.1 \\
Age (years) & $25-35$ & 48 & 68.6 \\
& $36-45$ & 16 & 22.9 \\
& $46-55$ & 5 & 7.1 \\
Educational qualification & $56-65$ & 1 & 1.4 \\
& Specialist & 31 & 44.3 \\
& Resident & 39 & 55.7 \\
\hline
\end{tabular}

Table 2 Awareness of participants regarding risk factors of retinopathy of prematurity and who performs screening for retinopathy of prematurity

\begin{tabular}{llll}
\hline Variable & Category & Frequency & Percent \\
\hline Causes of ROP & Low gestational age & 2 & 2.9 \\
& Weight less than 1800 g & 5 & 7.1 \\
& Sick require oxygen & 2 & 2.9 \\
& All the above & 60 & 85.7 \\
& Don't know & 1 & 1.4 \\
Eye test to be & Retina specialist & 21 & 30.0 \\
performed By & Pediatric ophthalmologist & 48 & 68.6 \\
& Don't know & 1 & 1.4 \\
\hline
\end{tabular}

and $6(8.6 \%)$ participants respectively. 22 (31.4\%) did not know how ROP is treated. In response to the question regarding the participants' opinion on how successful the treatment of ROP is in preventing blindness, only 26 (37.1\%) thought it is good, 17 (24.3\%) said very good, 26 (37.1\%) considered it satisfactory, while only one (1.4\%) thought it is poor.

Only $16(22.9 \%)$ pediatricians were satisfied with their current status of awareness and knowledge on ROP, while $30(42.9 \%)$ were not satisfied, $24(34.3 \%)$ had no idea about the current status on the awareness and knowledge.

\section{Discussion}

Retinopathy of prematurity, when severe can result in permanent visual disability, causing a high financial burden for the community and the individual. Guidelines have been established in some countries to enhance early identification and prompt treatment of babies with ROP. Pediatricians who are the primary caregivers of premature babies ought to be aware of the risk factors, screening and referral protocols, as well as treatment modalities and outcomes. This will lead to improvement in the quality of care of these babies, and thus saving their sight, along with the inherent costs of blindness to the individual and community [13].

In our study, although the majority of pediatricians had knowledge about some of the risk factors for ROP, a significant number of them were not aware of the correct timing of ROP screening. This may be due to the lack of clear established screening protocols for ROP in

Table 3 Awareness of participants regarding timing of ROP screening

\begin{tabular}{llll}
\hline Variable & Category & Frequency & Percent \\
\hline Period for & 4-6 weeks & 41 & 58.6 \\
first eye test & Depend on the gestational age & 25 & 35.7 \\
& Don't know & 3 & 4.3 \\
& Other & 1 & 1.4 \\
\hline
\end{tabular}


Palestine, and so it may contribute to a delayed detection of the problem.

The American Academy of ophthalmology clarified that indications for screening are region dependent. Thus, it is advisable to develop more researches about possible risk factors and screening criteria specific to country, instead of relaying merely on the criteria published by developed countries. Gilbert [3] advised those who did not have local screening guidelines for ROP to use wide screening criteria.

Identifying barriers for referral is vital for formulating any successful model. In our study the major reason given for not screening for ROP was the unavailability of an ophthalmologist in the same hospital according to over a third of the participants. In a study done by Mohammad et al. from the United States about barriers for ROP screening or follow-up eye care after discharge from the NICU, it was found that infants who were not screened for ROP in the NICU had a greater risk for missing follow-up eye care compared to those who had their first retinal examination in the NICU [18]. This highlights the importance of screening taking place in the NICU and disseminating information about ROP in our hospitals.

Unfortunately, some of the participants in our study were sure that ROP is not treatable. This may affect their willing to refer cases for screening, and thus prevents early detection and treatment of such a preventable issue.

The role of the nursing staff is critical to a successful prevention of ROP- induced blindness as they help in monitoring oxygen saturation targets during the whole period of treatment. They are responsible for feeding and temperature control and play a vitally important role in preventing late sepsis, they participate actively in the recommendations to the patient's family regarding follow up examinations after discharge [19].Apart from this, many cases of prematurity are babies of multiple gestations after IVF as their parents complained of infertility for years, so there might be a need to raise awareness among obstetricians and parents who are contemplating IVF as well.

In our study, only $16(22.9 \%)$ pediatricians were satisfied with their current status on awareness and knowledge on ROP. Up to now, there are no studies concerning KAP for ROP among pediatricians published in Palestine. We hope this study to be a step in enhancing knowledge among pediatricians, as well as establishing clear protocols concerning referral and screening of ROP.

Our study had some limitations, as with all self-reported questionnaires, the data could not be validated as their practices were not observed. It did not include other health care providers i.e. nurses in the NICU, Also a question about the place where the babies are usually screened was not included in the questionnaire.
In order to improve the awareness of ROP among pediatricians, nurses, obstetricians, and parents we recommend appropriate coordination between pediatricians and ophthalmologists, as well as dissemination of information through publishing articles and seminars in medical conferences, medical journals and health education materials for parents so local multidisciplinary workshops to highlight this issue is recommended.

\section{Conclusion}

The study findings suggest that a large majority of specialists and residents were aware of ROP as a disease, but had less information about ROP screening guidelines, service delivery and treatment modalities. The study suggests the need to create close coordination between pediatricians and ophthalmologists to address screening guidelines and barriers for service delivery in our country. The vital role of pediatricians in any screening program supports the need to enlighten them and increase their awareness by dissemination of information about ROP through seminars and literature, as well as spreading awareness among key members involved in preterm baby care.

\section{Abbreviations \\ CA: Chronological age; GA: Gestational age; KAP: Knowledge, attitude, and practice; NICU: Neonatal intensive care unit; PMA: Postmenstrual age; ROP: Retinopathy of prematurity}

\section{Acknowledgments}

The Authors would like to thank all participants without whom there would be no study.

\section{Funding}

This work has been entirely been made by the efforts and the financial support of the authors. No fund or financial assistance has been provided from other resources.

\section{Availability of data and materials}

These data represent doctors working in hospitals and based on the ethical approval from An-Najah National University, IRB ethical committee, data will be kept confidential and we can only submit the findings.

\section{Authors' contributions \\ MA has made substantial contributions to the conception and design of the study, the acquisition of data, the analysis and interpretation of the data, and the drafting of the manuscript. JQ has made substantial contributions to design, data analysis and interpretation, and to drafting the manuscript. $\mathrm{HI}$ has made substantial contributions to the conception and design of the study, the acquisition of data, the analysis and interpretation of the data, and the drafting of the manuscript. LY has made substantial contributions to the conception and design of the study, the acquisition of data, the analysis and interpretation of the data, and the drafting of the manuscript. All authors have given final approval of the version to be published.}

Ethics approval and consent to participate

Potential participants were approached by the researchers after ethical approval had been granted by An-Najah National University, IRB ethical committee.

Consent for publication

The manuscript doesn't contain any individual person's data in any form. 


\section{Competing interests}

The authors declare that they have no competing interests.

\section{Publisher's Note}

Springer Nature remains neutral with regard to jurisdictional claims in published maps and institutional affiliations.

\section{Author details}

${ }^{1}$ Department of Special Surgeries, Faculty of Medicine and Health Sciences, An-Najah National University Hospital, An-Najah National University, Nablus, Palestine. ${ }^{2}$ An-Najah National University, Nablus, Palestine.

Received: 25 April 2017 Accepted: 7 August 2018

Published online: 13 August 2018

\section{References}

1. Terry TL. Extreme prematurity and fibroblastic overgrowth of persistent vascular sheath behind each crystalline lens: I. Preliminary report. Am J Ophthalmol. 1942;25(2):203-4.

2. Waheeb S, Alshehri K. Incidence of retinopathy of prematurity at two tertiary centers in Jeddah, Saudi Arabia. Saudi J Ophthalmol. 2016;30(2):109-12.

3. Gilbert C. Characteristics of Infants with Severe Retinopathy of Prematurity in Countries with Low, Moderate, and High Levels of Development: Implications for Screening Programs. Pediatrics. 2005;115(5) https://doi.org/ 10.1542/peds.2004-1180

4. Kim TI, Sohn J, Pi SY, Yoon YH. Postnatal risk factors of retinopathy of prematurity. Paediatr Perinat Epidemiol. 2004;18(2):130-4.

5. Akkoyun I, Oto S, Yilmaz G, Gurakan B, Tarcan A, Anuk D, Akgun S, Akova YA. Risk factors in the development of mild and severe retinopathy of prematurity. J AAPOS. 2006;10(5):449-53.

6. Abdel HA, Mohamed GB, Othman MF. Retinopathy of prematurity: a study of incidence and risk factors in NICU of Al-Minya University Hospital in Egypt. J Clin Neonatol. 2012;1(2):76.

7. Fierson WM, Saunders RA, Good W, Palmer EA, Phelps D, Reynolds J, Chiang MF, Ruben JB, Granet DB, Blocker RJ, Bradford GE. Screening examination of premature infants for retinopathy of prematurity. Pediatrics. 2013;131(1):189-95.

8. Section on Ophthalmology American Academy of Pediatrics, American Academy of Ophthalmology; American Association for Pediatric Ophthalmology and Strabismus. Screening examination of premature infants for retinopathy of prematurity. Pediatrics. 2006;117(2):572-6.

9. Wilkinson AR, Clark D, Fielder A, Marlow N, Schulenburg WE, Weindling AM. Retinopathy of prematurity: guidelines for screening and treatment. The report of a joint working party of the Royal College of Opthalmologists and the British Association of Perinatal Medicine. Early Hum Dev. 1996:46(3):239-58.

10. Jefferies AL. Retinopathy of prematurity: recommendations for screening Paediatr Child Health. 2010;15(10):667.

11. Mutlu FM, Sarici SU. Treatment of retinopathy of prematurity: a review of conventional and promising new therapeutic options. Int J Ophthalmol. 2013;6(2):228.

12. Sathiamohanraj SR, Shah PK, Senthilkumar D, Narendran V, Kalpana N Awareness of retinopathy of prematurity among pediatricians in a tier two city of South India. Oman J Ophthalmol. 2011;4(2):77-80

13. Uhumwangho OM, Israel-Aina YT. Awareness and screening for retinopathy of prematurity among paediatricians in Nigeria. J West Afr Coll Surg. 2013;3(3):33.

14. Patwardhan SD, Azad R, Gogia V, Chandra P, Gupta S. Prevailing clinical practices regarding screening for retinopathy of prematurity among pediatricians in India: a pilot survey. Indian J Ophthalmol. 2011;59(6):427.

15. Blencowe H, Lawn JE, Vazquez T, Fielder A, Gilbert C. Preterm-associated visual impairment and estimates of retinopathy of prematurity at regional and global levels for 2010. Pediatric Res. 2013;74(S1):35.

16. Rani PK, Jalali S. Knowledge, attitude, practice study of retinopathy of prematurity amongst pediatricians attending a neonatal ventilation workshop in South India. World J Retina Vitreous. 2011;1:9-13.

17. Jalali S, Anand R, Kumar H, Dogra MR, Azad R, Gopal L. Programme planning and screening strategy in retinopathy of prematurity. Indian J Ophthalmol. 2003;51(1):89.

18. Attar MA, Gates MR, latrow AM, Lang SW, Bratton SL. Barriers to screening infants for retinopathy of prematurity after discharge or transfer from a neonatal intensive care unit. J Perinatol. 2005;25:36-40.

19. Kalyan G, Moxon S. The role of neonatal nurses in the prevention of retinopathy of prematurity. Indian Pediatr. 2016;53(Suppl.2):S143-50.

Ready to submit your research? Choose BMC and benefit from:

- fast, convenient online submission

- thorough peer review by experienced researchers in your field

- rapid publication on acceptance

- support for research data, including large and complex data types

- gold Open Access which fosters wider collaboration and increased citations

- maximum visibility for your research: over $100 \mathrm{M}$ website views per year

At $\mathrm{BMC}$, research is always in progress.

Learn more biomedcentral.com/submissions 